\title{
INCIDENCIA DE LA REGULACIÓN DEL MODELO ESTÁNDAR DE CONTROL INTERNO EN LAS ENTIDADES DEL SECTOR PÚBLICO EN COLOMBIA $^{1}$ \\ Incidence of the regulation of the standard internal control model in public sector entities in Colombia.
}

\author{
Hasbleidy Vargas Toledo \\ Universidad Santo Tomas. \\ Correo electrónico: hasbleidyvargas@usantotomas.edu.co
}

${ }^{1}$ Este texto surge a partir del proyecto que fue realizado como opción de grado para optar por el titulo de Contadora

Pública de la Universidad Santo Tomas.

\begin{abstract}
Resumen
A través de la presente investigación se pretende identificar la incidencia de la regulación del Modelo Estándar de Control Interno en las entidades del sector público en Colombia, a partir de la comparación de los manuales de aplicación. Siguiendo este propósito, en este estudio se implementó tanto una metodología de carácter cualitativo, la cual implicó un proceso de triangulación de la información obtenida a partir de una revisión bibliográfica y normativa, como la aplicación de entrevistas semiestructuradas. A modo conclusivo, se considera que los objetivos de la actualización del manual sobre MECI no se cumplen; por lo tanto, se podría afirmar que sería más adecuado un modelo mixto para las empresas colombianas.
\end{abstract}

Palabras clave: Entidades públicas, modelo estándar de control interno, normativa.

\begin{abstract}
The purpose of this research is to identify the impact of the regulation of the Internal Control Standard Model in public sector entities in Colombia, based on the comparison of the application manuals. Following this purpose, a qualitative methodology was implemented in this study, which implied a triangulation process of the information obtained from a bibliographic and normative review, as well as the application of semi-structured interviews. In conclusion, it is considered that the objectives of updating the $\mathrm{MECl}$ manual are not met; therefore, it could be stated that a mixed model would be more appropriate for Colombian companies.
\end{abstract}

Key words: Public entities, standard internal control model, regulations. 


\section{Introducción.}

En los comienzos de la historia Contable se han presentado diferentes formas de conocer el control en los negocios, a través de distintos mecanismos que ayudan a vigilar la transparencia de los procesos. Sin embargo, lo anterior no ha sido suficiente para evitar algún tipo de fraude dentro de una organización, en tanto dichos fraudes se pueden presentar en los diferentes movimientos financieros que realizan las entidades.

Ahora bien, desde los principios del aseguramiento de la información internacional se pretende que la información del profesional contable sea verídica y que se cumpla con el objetivo de que el usuario que requiera la información pueda confiar en ella; lo anterior, con el fin de que puedan ser tomadas buenas decisiones, proporcionando criterios que son utilizados para el mejoramiento de diferentes procesos y procurando evitar el riesgo de fraudes o pérdidas financieras, dado que durante años se han presentado diferentes casos y sectores de fraude. Sin embargo, hay que decir que estos últimos a su vez han sido considerados muy importantes para la evolución de nuevos mecanismos de prevención y de vigilancia, en países con grandes intereses económicos (Florian, 2016).

Así mismo, se entiende que las actividades que realizan las empresas a nivel interno son vitales para el desarrollo económico. La contabilidad también se considera esencial en tanto desde esta se genera información de suma importancia y que puede resultar útil para diferentes fines, además de garantizar que las actividades internas de las empresas se realicen a cabalidad; sin embargo, se requiere de un mecanismo de evaluación y de administración interna que sea eficiente y que le permita a las empresas cumplir con sus objetivos, respaldando la información proporcionada por los profesionales contables. Valga decir que dicho mecanismo ha evolucionado con el tiempo y en la actualidad se denomina Control Interno (Acevedo, Castro, \& Figueroa, 2005); este, a su vez es definido como un sistema integrado por un conjunto de acciones realizadas con el fin de fortalecer el crecimiento de cada entidad, en cumplimiento de sus objetivos y capacidades (García, 2014).

Por otro lado, con el propósito de verificar la información que se debe proporcionar desde la contabilidad, mediante la validación y el aseguramiento que se espera de los procesos, los profesionales deciden respaldar y acompañar esta revisión interna con la auditoría, la cual es un mecanismo internacional adoptado por diferentes países para analizar y proporcionar información relacionada con los fenómenos financieros externos e internos, para el buen uso y control de los procesos contables (Sandoval, 2012).

Por su parte, las Normas Internacionales de Auditoría (NIA) fueron diseñadas por la Federación Internacional de Contadores (IFAC), la cual está encargada de fortalecer la profesión contable en el mundo (Barral, Bautista, \& Sánchez, 2014). Las diferentes áreas en las que se puede desempeñar la 
contabilidad dentro de una empresa permiten que la información que entrega un profesional sea real para la toma de decisiones. No obstante, es necesario que se desarrollen nuevos mecanismos para el cumplimiento de los objetivos de las entidades; lo anterior, con el fin de mantener los intereses económicos y financieros de las empresas. A su vez, brindando una visión diferente a la profesión, la IFAC pretende que, con las Normas de Aseguramiento, se desarrollen nuevos mecanismos que determinen y verifiquen la veracidad de la información contable.

Asu vez, debido a los diferentes fraudes financieros que han tenido lugar en distintas entidades en el mundo a lo largo de la historia actual, se ha desencadenado la necesidad de mantener el control interna y externamente en una empresa (Galaz, Yamazaki, \& Ruíz, 2015). Lo anterior, en tanto se considera que es indispensable para la buena toma de decisiones, implementar mecanismos que ayuden a identificar la causa de dichos fraudes en las diferentes áreas contables, minimizando el riesgo y administrandolo para generar confianza en las futuras actividades laborales.

Del mismo modo, se podrían crear modelos de aseguramiento financiero que permitan ajustar los objetivos de una entidad, conduciéndola a la implementación de resultados efectivos y eficientes, dentro y fuera de un mercado, siendo este el principio de la creación de más modelos de Control Interno, con características en diferentes sectores contables, administrativos, económicos entre otros. Estos a su vez han de ser implementados para demostrar la fiabilidad, la verificación de la contabilidad y la auditoría, en validación de la información proporcionada por el profesional contable (Quinaluisa, Ponce, Muñoz, Ortega, \& Pérez, 2018).

Dentro de las actualizaciones de estos modelos países como Colombia ponen en práctica la auditoría; los avances y los comienzos de su aplicación pondrían en marcha una profesión, a partir de la que se desempeña el cargo como analista de los fenómenos contables, el cual es el cargo más importante del país, y el que además se conoce como Revisor Fiscal. Este rol se desarrolló a partir del siglo XI en el segundo viaje de Colón, y a partir del mismo se designaba a una persona para controlar y para analizar el manejo de los fondos y de los bienes de la expedición, con el fin de generar control y de presentar informes adecuados cuando se arribaba al reinado católico. Sin embargo, hay que indicar que todo lo mencionado antes no debe dejar de lado la idea de que todas estas funciones son necesarias para generar fe pública sobre las operaciones (Florian, S.F).

Cabe resaltar, además, que desde 1931 en Colombia se desarrolló una estructura legislativa que estableció el rol de revisor fiscal. En la misma línea, con el Decreto 410 de 1971, en el Artículo 203, se define qué tipo de entidades son obligadas a contratar un profesional en el área anteriormente mencionada. Por otra parte, para el año 2005 el país adoptó un nuevo modelo de control denominado Modelo Estándar de Control Interno (MECI), el cual proporcionó una estructura de control, además de la gestión y la evaluación de las entidades del Estado; su propósito principal ha sido orientar a estas entidades hacia el cumplimiento de sus objetivos institucionales, así como definir la contribución de estos a los fines esenciales del Estado (DAFP, 2005): Dicho modelo fue 
puesto en marcha a partir del Decreto 1599 de 2005, el cual mencionaba, a través de sus artículos, la adaptación del modelo en las entidades del sector público, así como las características para su aplicación.

A partir del mencionado decreto, el Departamento Administrativo de la Función Pública [DAFP] diseñó el primer Manual Técnico del Modelo Estándar de Control Interno (MECI 1000:2005), en el cual se explican los instrumentos necesarios para la aplicación y desarrollo de cada uno de los elementos, componentes y subsistemas que tiene el modelo (DAFP, 2005).

Frente a lo anterior, Mancipe (2014) afirma que en su primera versión y dentro la regulación colombiana, $\mathrm{MECI}$ no era eficiente para las empresas del sector público y, por ende, hace alusión a las expectativas frente a su actualización en el año 2014.Al respecto de lo dicho, en su estudio la autora pretende presentar su propia visión sobre un modelo diferente, así como su alcance frente a los propósitos y a los objetivos de cada entidad. Sin embargo, sobre la actualización del manual no hay estudios que enfaticen lo práctico, lo útil, las ventajas o las desventajas del modelo.

Por otra parte, el mecanismo de Control Interno es conocido como uno de los mejores modelos que se pudo impulsar en Colombia, con el fin de tratar de administrar y de disminuir el riesgo o el fraude en las operaciones contables y financieras, garantizando la eficiencia de las operaciones, así como la eficacia y la confiabilidad de la información; de igual modo, con el fin de que su verificación sea adecuada, prudente con los procesos y que cumpla con los objetivos de la entidad.

Así las cosas, el presente artículo investigativo comprende la importancia de la normatividad y la incidencia de la regulación de los organismos de control, como aspectos aplicadoss a las entidades del estado, reflejando la importancia de la aplicación de un modelo de Control Interno, a partir del cumplimiento de la normatividad ofrecida por el DAFP y el gobierno colombiano, en la tabla 1 se muestran los objetivos del presente trabajo.

Tabla 1.

Objetivos del Modelo Estándar de Control Interno en las entidades del sector público en Colombia.

Objetivo general: Analizar la incidencia de la regulación del Modelo Estándar de Control Interno en las entidades del sector público en Colombia del 2005 al 2014.

Objetivos específicos : 1. identificar la regulación del Modelo Estándar de Control Interno en las entidades del sector público en Colombia del 2005 al 2014.

2. Comparar el Manual Técnico del Modelo Estándar de Control Interno del 2005 al 2014.

3. Validar la implementación del Manual Técnico del Modelo Estándar de Control Interno con entidades del sector público a través de entrevistas semiestructuradas con expertos profesionales. 
Para finalizar este apartado es necesario indicar que, como se ha mencionado antes, este articulo investigativo se orienta al análisis de la incidencia de la regulación del $\mathrm{MECl}$ en las entidades del sector público en Colombia. Con este propósito, se identificó la regulación del modelo, comprándose con el MECI 1000:2005 y su actualización en el 2014, validando la implementación del manual, regulada por el DAFP.

A continuación, en el siguiente apartado se describirá el propósito y la metodología seguida para este estudio.

\section{Metodología.}

Este trabajo de investigación se desarrolló bajo el enfoque cualitativo siguiendo los planteamientos de Gómez, Sánchez y Diaz (2015), y se encuentra orientado por el paradigma hermenéutico. Además, se asume como propósito adelantar un análisis de la incidencia de la regulación del Modelo Estándar de Control Interno en las entidades del sector público en Colombia, desde el año 2005 hasta el año 2014. Por su parte, este estudio es de carácter exploratorio, con un alcance descriptivo.

En el diseño de la investigación se consideraron los protocolos definidos por Trucco, Gómez, Fajardo y Facundo (2016). Así las cosas, en primer lugar se realizó una revisión bibliográfica, cuyos resultados fueron contrastados; en segundo lugar, se realizó una revisión normativa y, en tercer lugar, se concluyó con una serie de entrevista semiestructurada, la cual fue dirigida a profesionales que se desempeñaban en el área de Control Interno de la ciudad de Bogotá. Con base en lo anterior, la investigación se desarrolló a partir de las fases que se describen en los siguientes párrafos a continuación.

Fase 1: se realizó una revisión bibliográfica, con el fin de analizar el material teórico sobre el Modelo Estándar de Control Interno en Colombia, utilizando los siguientes criterios de búsqueda: Control Interno, Modelos de Control Interno, COSO y MECl. A partir de la revisión bibliográfica se pudo identificar el proceso del MECI en Colombia; su aplicación y sus características. La revisión bibliográfica se realizó particularmente as través de la plataforma de Google Académico, en tanto en las bases de datos especializadas no se encontraron documentos sobre MECI. Por su parte, el periodo de tiempo contemplado fue a partir de la promulgación del Decreto 410 del año 1971 y hasta el año 2020; sin embargo, no se encontraron documentos relacionados con fecha posterior al año 2015.

Fase 2: se revisó la normatividad nacional e internacional relacionada con los diferentes modelos de Control Interno, con el fin de aterrizar al contexto colombiano el Modelo Estándar de Control Interno aplicado.

Fase 3: con base en las fases anteriores se elaboró un guión para la realización de tres entrevistas semiestructuradas, las cuales fueron dirigidas a tres expertos académicos y profesionales de la ciudad de Bogotá, con experiencia en el área de Control Interno. Es importante mencionar que, 
para esta fase, se realizó una prueba piloto con el fin de confirmar la pertinencia de las preguntas; así mismo, es necesario indicar que se analizaron las respuestas de cada entrevistado, las cuales fueron analizadas tomando un tiempo estimado, y a partir de la información correspondiente de cada una de ellas, con el fin de encontrar una conexión entre la normatividad y la información obtenida.

Para finalizar, se realizó una triangulación de la información obtenida tanto a partir de la revisión bibliográfica, como de la revisión normativa y de las entrevistas; lo anterior permitió generar resultados a partir de categorías de análisis, los cuales serán presentados a continuación.

\section{Resultados}

Reconocimiento inicial del Control Interno.

A través de los años la contabilidad se ha alimentado de grandes antecedentes históricos. A partir de estos han sido creados diferentes tipos de emprendimientos que generan riquezas. Lo anterior se ha realizado bajo el concepto de posesión, el cual implícitamente ha proporcionado la necesidad de Control. En la esfera de la contabilidad, el primer planteamiento que generó control fue la partida simple, describiéndose esta como una sola partida registrada en las operaciones contables; a su vez, dichos procesos han sido importantes dentro de la historia, para la contribución de la continuidad en un emprendimiento o de un negocio en marcha. De igual manera, los cambios que se han tenido en consideración, como principio contable, tratan de generar orden a los primeros movimientos financieros, que se presentaban en una organización (Acevedo, Castro, \& Figueroa, 2005). Así las cosas, la contabilidad ha sido considerada como una de las actividades más importantes en una empresa, en tanto brinda seguridad y eficacia al proceso de transparencia económica, política y social, proporcionando veracidad en la información que requiere un gerente, para efectuar una buena toma de decisiones (Flores \& Ibarra, 2006).

Por su parte, la modernización por la que han tenido que pasar las diferentes empresas, y la verificación de las actividades contables, han adelantado un organismo de seguridad de protección para aumentar la eficiencia en las operaciones (Cano, 2008); ello con el propósito de crear un sistema de Control Interno que es necesario para todo tipo de empresa; así mismo, desde los mecanismos de auditoría se ha buscado aumentar la rentabilidad proporcionando ayuda dentro de ella, con un aseguramiento de ocurrencia mínima del riesgo, desde lo que se pretende cumplir con los objetivos de cada entidad, evaluando y mejorando la eficiencia de sus procesos de gestión, así como cumpliendo con la normatividad establecida y con los estándares internacionales (The Institute of Internal Auditors, S.F).

\section{Contexto internacional del Control Interno.}

Durante el reconocimiento histórico del Control Interno se ha evidenciado la necesidad de un mecanismo para el buen funcionamiento y para el buen manejo de una empresa (Del Toro, 
Fonteboa, Armada, \& Santos, 2005).En su contexto internacional, el Control Interno es aplicable interna o externamente, en los diferentes sectores de una empresa. Como referencia de modelos que puede proporcionar este aseguramiento financiero, se evalúa y se encuentran los acuerdos más importantes en el sector internacional, los cuales son capaces de suplir los vacíos financieros que generaron las implementaciones erradas de procesos de vigilancia y control. Por su parte, desde Ios Tratados de Basilea I, Basilea II y Basilea III, los cuales fueron redactados en Suiza, se discute el Control Interno del sistema financiero; estos a su vez son presentados a causa de las crisis financieras que ha tenido lugar a nivel mundial, ayudando a las operaciones de moneda extranjera. Así bien, Basilea es aplicable con el fin de restaurar la confianza y la estabilidad dentro del sistema internacional (Fuentes, S.F; Galaz, Yamazaki, \& Ruíz, 2015).

Es necesario aclarar que se manifiesta la importancia de los acuerdos de Basilea, en tanto se conciben como el punto de partida para la creación y para la aplicación de muchos más modelos en distintos campos contables y enen diferentes empresas en el mundo, llegando a la creación del modelo Committee of Sponsoring Organizations of the Treadway Commission (COSO) de los Estados Unidos, el cual es uno de los modelos más robustos en el sistema de Control Interno. Este fue creado bajo el marco conceptual de Control para su aplicación, e integra diferentes definiciones sobre Control Interno (Quinaluisa, Ponce, Muñoz, Ortega, \& Pérez, 2018).

Dentro del Informe COSO (1992) el Control Interno se define de la siguiente manera:

Un proceso, efectuado por el Consejo de la Administración, la Dirección y el resto de personal de una Entidad, diseñado para proporcionar una razonable seguridad con miras a la realización objetivos en categorías como; la Efectividad y eficiencia de las operaciones; Confiabilidad de la información financiera y Acatamiento de las leyes y regulaciones aplicables (Informe COSO Committee of Sponsoring Organizations of the Treadway, 1992, p. 4).

A su vez, COSO fue diseñado bajo los criterios y las necesidades que identificaron cinco representantes de organizaciones privadas, tomando las bases fundamentales de la auditoría, como proceso integrado a las operaciones de las empresas (Quinaluisa, Ponce, Muñoz, Ortega, \& Pérez, 2018): Dicho modelo también permite desarrollar diferentes procesos operativos de carácter administrativo y de negocios, apoyando siempre, desde su elaboración, la toma de decisiones y el gobierno corporativo de las diferentes organizaciones (Committee of Sponsoring Organizations of the Treadway, 2013).

Dentro del resumen que presenta la traducción al idioma español del Marco Integrado de Control Interno, realizado en conjunto por las diferentes asociaciones privadas de auditoría, se define la evaluación de riesgos. Estos por su parte permiten juzgar el peligro de que los errores señalados no alcancen a llegar a los estados financieros, lo cual incide en que la contabilidad pueda entrar como primera instancia de conocimientos de los procesos para complementar y para observar las nuevas 
técnicas de evaluación que tenía la auditoría, siendo esto ejecutado por el modelo.

Algunos de los modelos de Control Interno antes mencionados son conocidos por su aplicación en temas financieros y entre ellos se encuentra Criteria of Control (COCO) de Canadá. Este modelo, que es llevado a cabo bajo la revisión de COSO, se caracteriza por la complejidad de los conceptos, por su lenguaje y por hacer posible el alcance total de Control Interno dentro de una entidad (Quinaluisa, Ponce, Muñoz, Ortega, \& Pérez, 2018).

Por otra parte, con modificaciones dentro del contexto internacional se encuentra el modelo Control Objectives for Information and related Technology (COBIT) de Australia, el cual se describe como una herramienta que proporciona seguridad cibernética; ello, en tanto a partir del mismo se considera que, para las entidades, la información que se encuentra alojada en los Software es muy valiosa, de tal manera que creen que es el activo más significativo que puede tener una entidad; a su vez, con ayuda de la evaluación de la administración de riesgos y la regulación que proporciona el modelo, consideran que pueden mitigar o evitar algún riego de información o algún tipo de fraude, dentro y fuera de las operaciones de negocios (Information Systems Audit and Control Assocition, 2007).

Otros modelos de Control Interno que también se han considerado importantes y reconocidos se pueden observar en la Tabla 2. Cabe resaltar que la aplicación de cada uno de estos modelos se realiza con el fin de proporcionar información a la contabilidad, en diferentes áreas, aumentando la seguridad de los datos que proporciona un profesional.

Ahora bien, de acuerdo con los modelos mencionados antes y tomando como referencia el contexto internacional, Control Interno es un proceso integrado a las operaciones, siendo adoptados en su quehacer organizacional los modelos que más le proporcionen seguridad de la información, los que a su vez crean la necesidad de acuerdo con su aplicación, que con base en su definición, sirven para la mitigación y para la evasión del riesgo financiero, cibernético o de ambiente laboral en control, dentro de las empresas; su regulación la realiza el mecanismo deAuditoría Interna, pretendiendo ser efectiva para el mejoramiento de las operaciones de negocios (The Institute of internal Auditors, S.F).

Tabla 2.

Modelos de Control Interno

\begin{tabular}{ll}
\hline Cadbury (Reino Unido) & Vienot (Francia) \\
\hline Peters (Holanda) & King (Sudafrica) \\
\hline Marco Integrador de Control Interno Latinoamericano (MICIL) & System Auditability and Control (SAC) \\
\hline
\end{tabular}

Fuente: elaboración propia a partir de Quinaluisa, Ponce, Muñoz, Ortega, \& Pérez (2018). 


\section{Implementación del Control Interno en Colombia.}

Respecto de la aplicación de los diferentes modelos de Control Interno, bajo los cambios que tuvieron lugar en el marco del contexto internacional, Colombia emitió la primera Ley de Control en el año 1993, la cual debía ser aplicada de forma inmediata en todas las entidades del Estado. A partir de dicha Ley se definió el Control de la siguiente manera:

Un sistema integrado por el esquema de organización y el conjunto de los planes, métodos, principios normas, procedimientos, mecanismos de verificación y evaluación adoptados por la entidad con el fin de procurar que todas las actividades, operaciones y actuaciones, así como la administración de la información y los recursos, se realicen de acuerdo con las normas constitucionales y legales vigentes dentro de las políticas trazadas por la dirección y en atención a las metas y objetivos previstos (Ley 83 de 1993, 1993).

Luego de la publicación de la Ley 87 (1993), los procesos técnicos contables de implementación contribuyeron en la formación contable que deben tenerlos profesionales; sin embargo, el manual no ha sido adoptado en su totalidad y el mismo termina ajustándose a las necesidades de los empresarios en Colombia, pero afectando a los profesionales contables.

Teniendo en cuenta lo anterior, el entrevistado 2 (comunicación personal, 06 de agosto del 2019) aclara que a partir de su experiencia profesional, considera que las actualizaciones y la regulación, producto de los estándares internacionales tienen una buena intención pero a su vez encuentra que, aquello que se quiere regular, se pierde por la obligatoriedad de la Ley que se impone en Colombia; en tal sentido, considera que los procesos de adaptación e implementación de la norma han sido ineficientes.

\section{Adopción de estándares internacionales de Control Interno en Colombia.}

De común acuerdo con la Agencia Presidencial de los Estados Unidos [USAID], Colombia adoptó COSO para la creación de un nuevo modelo de Control Interno. Luego de la revisión por parte del gobierno colombiano, en el año 2005 se emitió el Decreto 1599, desde el cual se explicó un modelo con características similares, que ayudaría a las empresas a cumplir con sus objetivos y a busca un beneficio económico; del igual modo se establecía que este modelo debería proporcionar seguridad en procesos financieros; además se conoció por primera vez el Modelo Estándar de Control Interno [MECl], aplicado solo a entidades del Estado, pero sin realizarle ninguna modificación a la definición o a las características del modelo COSO. El plan que realizaron para la aplicación de MECI hizo que el modelo fuese viable para Colombia, un modelo estandarizado para cumplir a cabalidad y en totalidad la Ley de Control (DAFP, 2005).

Con el aval del Decreto 1599 (2005)proporcionado por el Congreso de la República se creó el Departamento Administrativo de la Función Pública [DAFP], como la entidad encargada de la regulación y de la emisión del $\mathrm{MECl}$, para entidades del Estado; a partir de ese momento se emitió el 
primer Manual Técnico del Modelo Estándar de Control Interno (MECI 1000:2005) (DAFP, 2005), en el cual se aclara que este tiene como finalidad proporcionar una estructura de gestión, evaluación y estrategia, dentro de las entidades del Estado, contribuyendo a fines esenciales (Tapia, Torres, \& Blanco, 2005). Así mismo, se planteó como propósito que las entidades encargadas de implementar este modelo tuvieran control en los procesos que desempeñaban, orientadas a los objetivos institucionales y a la contribución de estos a los fines esenciales del Estado (DAFP, 2005). De igual modo, dentro de MECI 1000 (2005) se estipuló, que desde dicha supervisión regulatoria, se tendría la potestad de solicitar a las empresas del Estado un informe auditor y una actualización anual, con el fin de verificar el buen uso del manual (DAFP, Decreto 943, 2014).

Ahora bien, se conoce que a partir de esta regulación, la implementación de este manual también ha tenido ciertas críticas, de tal modo que se ha opinado que la derogación de un decreto tras otro y la lenta aplicación de ellos, produce que la dinámica se ineficiente y responsable de que el proceso no sea comprensible, pues en medio de su aplicación, la labor profesional tiende a ser pausada, por las actualizaciones de las normatividades impuestas por el Estado. También se refleja que en el desarrollo de las actualizaciones de los decretos, la revisión de estos procesos, por parte de las entidades supervisoras es tardía, desconociendo los desarrollos correspondientes al MECI que durante años se han llevado a cabo (Mancipe, 2013) y que se implementan nuevas maneras de regulación, evidenciando que no hay una evaluación como la que se espera, y que la regulación y observaciones del DAFP no generan una posible actualización del MECI (DAFP, 2005).

Así mismo, la imposición de la regulación general ocasiona cierta incertidumbre para los profesionales que aplican los decretos en una empresa. Frente a estas falencias, el entrevistado 1 (comunicación personal, 27 de enero del 2019) alude a cierta comparación oportuna respecto de las las actualizaciones que ha tenido el modelo en relación con los manuales, tal como se puede leer a continuación:

Digamos que los manuales más allá de que sean buenos o malos corresponden a una realidad organizacional, seguramente la primera versión tenía su, nivel de complejidad, pero han venido actualizando de acuerdo a las necesidades de acuerdo a las comprensiones que las instancias de que les compete deben tener; puedo decir que un comparativo, de cómo ha cambiado sencillamente corresponde a las realidades y necesidades de las organizaciones colombianas del orden público(Entrevistado 1, comunicación personal, 27 de enero del 2019).

Más allá de generar culpas o de que la responsabilidad del DAFP se vea afectada, el entrevistado 1 (comunicación personal, 27 de enero del 2019) comprende que la eficiencia fundamental del MECI no está en el manual, si no en la disposición que el profesional le imprima a su labor.

Además, el entrevistado 3 (comunicación personal, 12 de diciembre del 2019) opina que la creación del $\mathrm{MECl}$ fue innecesaria, dado que su implementación es una adopción a las características que 
tiene COSO. Con respecto a este último modelo, el cual ya ha sido aceptado por las empresas del sector privado en Colombia, el entrevistado 3 (comunicación personal, 12 de diciembre del 2019) comenta que su aplicación podría ser inclusiva en entidades del sector público.

\section{Actualización de la normatividad colombiana en el sector público.}

Luego del reconocimiento inicial y de la publicación del primer documento instructivo propuesto (MECl 1000:2005) surgió el Decreto 943 (2014), desde el cual se estipuló la primera actualización de $\mathrm{MECl}$. Esta a su vez integró los valores que respaldan este modelo y planteó al servidor público como parte fundamental para el cambio, reemplazando por completo el esquema y las características desarrolladas en el primer manual y del mismo modo contemplando la autonomía del servidor público para el buen manejo de dicha actualización (Mancipe, 2014).

En cuanto a la actualización que ha tenido la normatividad colombiana en el sector público, autores como Méndez, Rueda \& Nuñez (2014) menciona lo siguiente:

Entre las principales novedades que trae la actualización del modelo, se encuentra que los tres subsistemas de control a los que se refería la versión anterior se convierten en dos Módulos de Control, que sirven como unidad básica para realizar el control a la planeación y la gestión institucional, y a la evaluación y seguimiento. A partir de esta modificación, la información y comunicación se convierten en un eje transversales al Modelo, teniendo en cuenta que hacen parte de todas las actividades de control de las entidades (Mendez, Rueda \& Nuñez, 2014, p.. 14).

El modelo mencionado permite identificar características importantes y busca siempre garantizar el cumplimiento de los objetivos institucionales de las entidades y la contribución a los fines del Estado (DAFP, 2014). Dentro de estas actualizaciones se identifica que las mismas no fueron adecuadas en su aplicación; al respecto, el entrevistado 1 (comunicación personal, 27 de enero del 2019) opina que al interior de las entidades del Estado las implementaciones se realizaron con rapidez.

yo veo es que la implantación ha sido muy rápida por lo menos en el distrito, entonces ha sido a través de auditorías internas que hemos venido interiorizando la importancia de entender estos dos pilares que he mencionado, como la administración del riesgo, y las líneas de defensa (Entrevistado 1, comunicación personal, 27 de enero del 2019).

Entre las principales falencias que el entrevistado 1 (comunicación personal, 27 de enero del 2019) encuentra, está la mala realización de las capacitaciones pertinentes para los servidores públicos, quienes en realidad no interiorizan el alcance de cada una de las características que ofrece el manual para su aplicación, afectando en el tiempo la implementación del manual en las entidades del Estado. Así mismo, una de las principales falencias que tiene el modelo de Control Interno es la ausencia una de regulación anual por parte del DAFP, en las entidades del Estado. 
En tal sentido, se han llegado a comprender las posibilidades y las capacidades que técnicamente puede proporcionar el MECI en Colombia, pero es necesario que se contemplen ciertas categorías, las cuales se entiende en esta investigación que han sido muy relevantes, y las que además fueron contrastadas con profesionales en el área de control y contabilidad con base a sus conocimientos, así como en el recorrido de su experiencia. Así bien, se entiende que es importante mencionarlas y comprender la importancia de un modelo sólido en Colombia, comenzando por la categoría referente a la incidencia que ha tenido el modelo, siendo la base principal en el sector público y de relevancia en el sector privado.

\section{Incidencia en el sector privado.}

Con el fin de preservar la esencia y la confianza en las entidades del Estado, la regulación es presentada dejando a un lado a las empresas del sector privado. Sin embargo, es importante que se tenga en cuenta a las mismas, de tal forma que estas cumplan con ciertas normativas relacionadas con un modelo de control: en la actualidad, las entidades privadas aplican el modelo COSO, el cual es la base de $\mathrm{MECl}$.

No obstante lo anterior, MECl es un modelo implementado en entidades del Estado, pero se ha identificado que estas entidades también tienen participación en inversiones como entidades privadas; esto quiere decir que las diversas implementaciones del modelo han sido adecuadas para un sector mixto. Al respecto, el entrevistado 1 (comunicación personal, 27 de enero del 2019) indica que en las empresas del sector privado ya existe un modelo el cual es COSO, y que es de libre albedrío su implementación, adicional a la normatividad nacional e internacional como la familia ISO, la cual esta siendo implementada por las empresas en Colombia:

La dimensión de Control Interno al modelo integrado de planeación de gestión a nivel de administración pública colombiano en todos sus niveles, desde el nivel Municipal, Departamental, Nacional, tiene particularidades porque hay unas regulaciones adicionales pero quien esté interesado en meterse en el ejercicio de Control Interno en el sector privado, tiene que sencillamente pues interesarse en el tema de capacitarse si se quiere en temas de esto que les cuento del enfoque COSO es un estándar internacional (Entrevistado 1, comunicación personal, 27 de enero del 2019).

Por su parte, al considerar la regulación que tiene el $\mathrm{MECl}$ en Colombia, siendo esta una de las primeras falencias que tiene para ser aplicado en el sector privado, se ha podido identificar que dentro del desarrollo de la labor de profesional, éste puede que sea inadecuado, teniendo en cuenta que la aplicación del modelo es desarrollada y actualizada para el cuerpo que compone las empresas del Estado en el sector público; por lo tanto el alcance de este modelo y la incidencia en las entidades privadas sería errada.

Asu vez, a través del modelo de Control Interno ha sido posible analizar las problemáticas del sector 
privado; por lo tanto, este fue diseñado en respuesta a los diferentes fraudes financieros que se habían presentado a nivel internacional, con el fin de identificar los mismos antes de que ocurrieran. Además, con el objetivo de facilitar el análisis y la identificación de estos fraudes, a través de dichos modelos es posible examinar el funcionamiento de las actividades en las entidades, indagando dentro de sus departamentos por los factores que puede ocasionar estafas, para evidenciarlas antes de que ocurran.

El modelo surge de una preocupación muy grande dadas las crisis económicas que surgen en determinado momento de la vida económica de Estados Unidos y el gobierno, como parte regulatoria, a través de la bolsa de valores de New York el hecho de que se propongan a través de formas en el compilado fue una firma de auditoría Deloitte and Touche, quien compiló conceptos básicos del modelo. Vienen de un espíritu empresario privado en donde lo que prevalece para la empresa privada, específicamente en Estados Unidos, es mantener la tasa de ganancia(Entrevistado 2, comunicación personal, 06 de agosto del 2019).

Lo anterior quiere decir que si el modelo proviene de actividades en sectores privados, podríamos plantearnos la posibilidad de aplicar MECl en estos sectores, examinando las causas que fueron planteadas para ser aplicadas específicamente en el sector público. Sin embargo, mediante la adopción y el estudio que hizo el gobierno colombiano en el año 2005 se aprobó la aplicación del modelo en las entidades del Estado.

\section{Modelo ideal para los dos sectores o un modelo mixto.}

Teniendo en cuenta las falencias que tiene el modelo en el sector público colombiano, se consultó la opinión de los entrevistados, sobre un modelo ideal o sobre la posibilidad de un modelo mixto. Con anterioridad se ha descrito el proceso de implementación del modelo en el sector privado y la adopción de ese modelo en el sector público en Colombia; también se ha especificado que este tipo de características servirían para un nuevo modelo de implementación mixto. Sin embargo, las actividades que desarrolla cada tipo de empresa son totalmente diferentes; esto querría decir que por órdenes del DAFP, como los encargados de la regulación y del diseño de los manuales, no se podría aplicar dicho modelo en los dos sectores.

A su vez, teniendo en consideración tanto el modelo COSO, el cual fue implementado en Colombia para el sector privado, así como la adopción que tomó el sector público para diseñar $\mathrm{MECl}$, además de las respuestas de los entrevistados, se puede concluir que este tipo de modelos servirían tanto para el sector público como para el sector privado. No obstante, el entrevistado 1 (comunicación personal, 27 de enero del 2019) cuestiona la propuesta de esta investigación y comenta que el enfoque del sector público es diferente al sector privado, en tanto los objetivos internos de las empresas privadas tienen una dirección diferente a los de las entidades públicas. En tal sentido, el entrevistado 1 (comunicación personal, 27 de enero del 2019) expone que no podría configurarse un modelo mixto o que finalmente un modelo de este tipo no sería el apropiado para las necesidades de 
control de cada sector, e identifica las falencias que se han presentado a través de los años en su implementación.

Por otra parte, teniendo en cuenta que las actualizaciones que realiza el DAFP no son las pertinentes para las actividades que las empresas del Estado desarrollan de manera continua, se considera que las aplicaciones de los profesionales en el área son incorrectas (Cárdenas, 2012), y que además hacen falta procesos de capacitación profesional, específicamente en lo que respecta a los manuales y a la normatividad que es expedida con regularidad (entrevistado 1, comunicación personal, 27 de enero del 2019).

Contrario a lo anterior, el entrevistado 2 (comunicación personal, 06 de agosto del 2019) plantea un modelo ideal que ofrezca calidad de resultados para los dos sectores, esto es un modelo mixto. Al respecto, resalta la importancia de los modelos de control interno implementados en Colombia para los diferentes sectores y entidades; no obstante, también plantea la necesidad de desarrollar un modelo propio, desde el cual los profesionales sean la clave para su implementación y para su revisión, con el fin de evitar fallas futuras. Así bien al respecto sugiere trasferir las características representativas de modelos como COSO o MECl, los cuales representan la calidad de las funciones en control interno, o modelos como COCO, el cual está orientado a las personas, al ambiente de trabajo dentro de una entidad y a planteamientos diferentes para el desarrollo de control interno en una entidad.

Además de las opiniones de los profesionales que se han citado anteriormente, el entrevistado 3 (comunicación personal, 12 de Diciembre del 2019) indica que se podría estudiar la posibilidad de una transición a un modelo que sea completo y robusto para las empresas privadas y públicas, asunto que él contempla como solución a la problemática que produce generar una actualización tras otra, respecto de la regulación normativa en Colombia; así las cosas considera a COSO como solución y ejemplo de un modelo que pueda cumplir con los objetivos dentro de una empresa de cualquier sector, aduciendo que MECI puede ser descartado.

\section{Conclusiones.}

En el presente trabajo se logró evidenciar que la aplicación del modelo estandarizado solo está dirigida hacia las entidades del Estado, pero aún no se cuenta con algún modelo aplicado para el sector privado, modelo que además sea de creación propia para el contexto de Colombia; por lo tanto, se considera que este estudio puede orientar procesos para futuras investigaciones, en las cuales se analicen cuestiones de este tipo.

Ahora bien, partiendo de la Ley 87 de 1993 (1993), la cual fue implementada en Colombia con el fin de fortalecer a las empresas, se pudo evidenciar la importancia del Control Interno en las organizaciones colombianas, y de la mejora en la calidad de las operaciones; teniendo en cuenta lo 
anterior, se consideró que era necesario un modelo como $\mathrm{MECl}$, el cual propendiera por mitigar, evaluar o administrar el riesgo que causan los procesos fraudulentos dentro de una entidad en el sector público (Entrevistado 3, 08, 2019). A su vez, se también se tuvo en cuenta la incidencia de la regulación que emite el gobierno nacional a partir de las actualizaciones que publica el DAFP; sin embargo, se considera la necesidad de una mayor precisión respecto del enfoque que puede tener el modelo en las empresas del Estado.

De igual manera, se comparó el modelo a partir del Decreto 1599 de 2005 (2005), desde el cual se presentó el primer Manual describiendo las características de $\mathrm{MECl}$, en contraste con las actualizaciones que presentó el Decreto 943 de 2914 (2014), lo cual permitió concluir que se han desarrollado cambios desde su estructura, hasta los elementos que componen el modelo, satisfaciendo las necesidades que se identificaron en el primer manual, para implementarlas en la última actualización. Con base en lo anterior, la actualización busca cumplir con el Marco Normativo que regula los Modelos de Control Interno en Colombia.

Por su parte, a fin de comprender los procesos de regulación en las empresas del sector público, se considera que las Juntas Directivas tienen mucho que ver, puesto que las empresas del Estado mantienen relaciones e inversiones en compañías privadas; a este tipo de negocios se les denomina economía mixta. Por lo anterior, en la entrevista que se propuso para este estudio, una de las preguntas más relevantes fue la relacionada con la posibilidad de un modelo mixto de control en Colombia. En estos procesos, la regulación del Estado no aplica obligatoriamente, porque estas inversiones que mantienen las empresas del sector público, esto es, su parte accionaria, no está obligada a ejercer procesos de control interno con MECl; lo anterior quiere decir que la normatividad para las empresas del sector público que tienen mayor capital accionario en el sector privado no rige.

Así mismo partiendo desde la estructura y el desarrollo del modelo que es enfocado a un tipo de inversión extranjera, $\mathrm{MECl}$ es una recopilación de COSO, siendo COSO un modelo enfocado a diferentes tipos de negocio, y desde el cual se busca identificar y evitar cualquier clase de fraude financiero de gran magnitud, lo cual puede considerarse como una "cultura" diferente a lo que se maneja en un país como Colombia (Entrevistado 2, comunicación personal, 06 de agosto del 2019).

Finalmente, en este estudio se identificó que la implementación de los decretos es ineficiente, pero estas deficiencias parten tanto desde el diseño y el modelo, como de su aplicación; esto último, dado que también se considera como una falencia la falta de conocimiento por parte de los profesionales que se encargan de realizar los procesos de control. Por lo tanto, es importante considerar la creación de un modelo que se pueda aplicar para una entidad con negocios de economía mixta, el cual proporcionaría seguridad financiera, protegiendo los intereses del Estado y los negocios de inversión de alto capital. En tal sentido, se considera que la realización de guías o de manuales que emite el DAFP serían innecesarias, si no se cuenta con un modelo de control estructurado, a partir del cual puedan ser abarcados los diferentes sectores económicos del país. 


\section{Referencias Bibliográficas}

Acevedo, P., Castro, M., \& Figueroa, C. (2005). Guía para evaluar el sistema de control interno bajo un enfoque del comité deorganizaciones patrocinadora (Committee of sponsoring organizations of the treadway commission-COSO), aplicable a la gran empresa del sector comercio de el salvador. (Tesis de pregrado, Universidad Dr. Jos é Matías Delgado). https://webquery.ujmd.edu.sv/siab/bvirtual/BIBLIOTECA\%20VIRTUAL/TESIS/01/CPU/ADCG0000859.p $\underline{\mathrm{df}}$

Barral, R. A., Bautista, M. R., \& Sánchez, M. H. (2014). Orientación Pedagógica sobre la aplicación de las Normas Internacionales de Auditoría (NIA). Publicaciones Consejo Técnico de la Contaduría Pública.

Cañas, C., Martinez, S., González, J., \& González, H. (2018). El revisor fiscal y sus obligaciones. COMISIONES TÉCNICAS. NIA, 1-6. https://incp.org.co/Site/publicaciones/info/archivos/El-revisor-fiscaly-sus-obligaciones-17012018.pdf

Cardenas, M. J. (2012). Evaluación del impacto del Modelo Estandar de Control Interno (MECI) como Herramienta de Gestión para algunas Entidades Públicas de la Ciudad de Palmira. Universidad Nacional de Colombia. http://www.bdigital.unal.edu.co/5766/1/7709006.2012.pdf

Committee of Sponsoring Organizations of the Treadway. (2013). Control Interno-Marco Integrado, resumen ejecutivo. PWC - AUDITORES INTERNOS. https://auditoresinternos.es/uploads/media items/cosoresumen-ejecutivo.original.pdf

Decreto 410 de 1971. Congreso de la república de Colombia 1971. file:///C:/Users/HP\%20NoteBook/ Downloads/Codigo+de+Comercio.pdf.

Decreto 1599 de 2005. (2005, mayo 20). Departamento Administrativo de la Función Pública. http://www.funcionpublica.gov.co/eva/gestornormativo/norma.php?i=16547

Decreto 943 de 2014. Departamento Administrativo de la Función Pública. Ministerio de Educación https://www.mineducacion.gov.co/cvn/1665/articles-347203_recurso_1.pdf

Departamento Administrativo de la Función Pública. (2005). Manual Técnico del Modelo Estándar de Control Interno. Obtenido de Manual Técnico del Modelo Estándar de Control Interno: http://apolo.uniatlantico.edu.co/SIG/MECl-1000-2005.pdf

Departamento Administrativo de la Función Pública.(2014). Manual Técnico del Modelo Estándar de Control Interno para el Estado Colombiano MECI 2014. Departamento Administrativo de la Función Pública de la Republica de Colombia. http://www.saludcapital.gov.co/CTDLab/Publicaciones/2015/MANUAL\% 20DEL\%20MECI\%202014\%20.pdf

Del Toro, J., Fonteboa, A., Armada, E., \& Santos, C. (2005). Programa de Preparación Economica para 
Cuadros. Material de consulta. Control Interno.. Centro de Estudios Contables Financieron y de Seguros. http://www.sld.cu/galerias/pdf/sitios/infodir/material_consulta_ci.pdf

Flores, Y., \& Ibarra, G. (2006). Diseño de control interno para medianas y pequeñas empresas. (Tesis de licenciatura, ICEA-BD-UAEH) https://repository.uaeh.edu.mx/bitstream/bitstream/handle/1234567 89/11007/Diseno\%20control\%20interno\%20pequena\%20y\%20mediana.pdf?sequence=1

Florian, C. (2016). La Auditoría, origen y evolución ¿por que en Colombia solo se conoce a través de leyes. Universidad Libre de Colombia.

Florian, C. (S.F). La auditoría, origen y evolución como se introduce en Colombia. Universidad Libre de Colombia.

Fuentes, G. (2014). El paso de Basilea II a Basilea III en la regulación financiera europea, aplicación al caso de Bankia. (Tesis de Maestría, Universidad de Valencia). https://riunet.upv.es/bitstream/handle/102 51/44402/Tesina\%20Basilea\%20III\%20-\%20Guillermo\%20Fuentes. pdf?sequence=1

Galaz, Yamazaki, \& Ruíz, U. (2015). COSO Marco de referencia para la implementación, gestión y control de un adecuado Sistema de Control Interno. Deloit.

García, M. (2014). El control Interno como mecanismo de transformación y crecimiento, una mirada desde la gestión gerencial de las empresas. https://repository.unimilitar.edu.co/handle/10654/12715

Gómez, C., Sánchez, V., Diaz, K. (2015). Analysis of Public Gender Policy in Colombia (1990-2014). Revista FACCEA, 5(2), 175-181

Committee of Sponsoring Organizations of the Treadway. (1992). Informe COSO, Committee of Sponsoring Organizations of the Treadway.

Intituto de Contabilidad y Auditor de Cuentas. (2011). Materialidad y Riesgo de Auditoría. Instituto de contabilidad y auditoría en cuentas, Ministerio de Economía y Hacienda Pública. http://www.icac.meh.es/Documentos/PUBLICACIONES/89.pdf

Information Systems Audit and Control Association. (2007). COBIT 4.1. Governance Institute. https://biblioteca.info.unlp.edu.ar/uploads/docs/cobit.pdf

Jefatura del Estado Europeo. (2015). Auditoría de cuentas. Boletín Oficial del Estado https://www.boe.es/buscar/pdf/2015/BOE-A-2015-8147-consolidado.pdf

Ley 83 de 1993. (1993, 3 de noviembre). Secretaria del Senado. http://www.secretariasenado.gov.co/senado/ basedoc/ley_0083_1993.html

Mancipe, L. (2014). ¿Por qué un nuevo MECI para el control público? En Contexto 2(2), 69-88. 
Mendez, A., Rueda, J. F., \& Nuñez, S. (2014). Manual Técnico del Modelo Estándar de Control Interno para el Estado Colombiano. Departamento administrativo de la Función Pública, República de Colombia. http://apolo.uniatlantico.edu.co/SIG/MANUALTECNICOMECI2014_7_7_2014.pdf

Quinaluisa, N., Ponce, V., Muñoz, C., Ortega, X., \& Pérez, J. (2018). El control interno y sus herramientas de aplicación entre COSO y COCO. Cofin Habana, 12(1), 268-283.

Resolución 357 (julio 23 de 2008) . Diario Oficial 47.071 de agosto 4 de 2008. (El Contador General de la Nación, Jairo Alberto Cano Pabón). https://www.defensoria.gov.co/public/Normograma\%20 2013_html/Normas/Res_357_2008_CGN.pdf

Sandoval, H. (2012). Introducción a la Auditoría. Red Tercer Milenio. http://www.aliat.org.mx/Bibliotecas Digitales/economico_administrativo/Introduccion_a_la_auditoria.pdf

Tapia, K., Torres, W., \& Blanco, Y. (2005). MECl 1000:2005. Actiweb.es. http://www.actiweb.es/ gestionpublica2010/historia.html

The Institute of internal Auditors. (2013). Normas Internacionales para el ejercicio profesional de la actividad deAuditoría Interna. The Institute of Internal Auditors.

Trucco, G., Gómez, C., Fajardo, M., Facundo, G. (2016). La corrupción en Latinoamérica y el ejercicio de la Profesión Contable. Revista FACCEA, 6(2), 170-176 